\title{
Qualitätskonzept als Herausforderung und Chance
}

\author{
Johannes Brühwiler ${ }^{a}$, Jean-Michel Gaspoz ${ }^{b}$, François Héritierc, Marc Müllerd, Lukas Zemp ${ }^{\mathrm{e}}$ \\ ${ }^{a}$ Leiter der Arbeitsgruppe Qualität SGAIM, ${ }^{b}$ Vorsteher des SGIM-Präsidiums, ${ }^{c}$ Präsident SGAM, ${ }^{d}$ Präsident MFE, \\ e Generalsekretär/Geschäftsstellenleiter SGIM
}

Die Schweizerische Gesellschaft für Allgemeine Innere Medizin SGIM und die Schweizerische Gesellschaft für Allgemeinmedizin SGAM haben beschlossen, mit den Arbeiten zur Gründung eines gemeinsamen Verbandes auch die Entwicklung eines Qualitätskonzeptes für die neue Fachgesellschaft SGAIM an die Hand zu nehmen. Das Thema Qualität bildet ein Kernelement für die neue Fachgesellschaft. Die Eigeninitiative ist zudem gefordert, weil die Idee eines zentralen Bundesinstitutes für Qualität in der Medizin auf breite Ablehnung gestossen ist.

Es wurde eine kleine Arbeitsgruppe eingesetzt," die innerhalb kurzer Zeit ein Konzept entwickelt hat, das nachfolgend vorgestellt wird. Dieses Konzept ist nun bei den beteiligten Gesellschaften in Vernehmlassung. Die Herausforderung liegt darin, dass die wesentlichen Elemente einer Qualitätsentwicklung vorliegen sollten, dass ein Nutzen für Patienten und Ärzte klar ersichtlich sein muss und ein messbares Element enthalten ist, das den Bestimmungen des KVG gerecht wird. In der Allgemeinen Inneren Medizin (AIM) spielen mehrfach erkrankte Patienten eine grosse Rolle. Es ist die Kernaufgabe, die unterschiedlichen krankheitsund organorientierten Sichtweisen in ein patientenorientiertes zielführendes Behandlungskonzept umzusetzen und das Ergebnis zu evaluieren.

\section{Bestandsaufnahme}

In einem ersten Schritt wurden die bestehenden laufenden Projekte zusammengetragen, die im erwähnten Kontext eine Rolle spielen. In allen sind Elemente der Qualitätssicherung und Entwicklung enthalten. Viele Projekte sind mit viel Engagement und exzellentem Fachwissen unterwegs. Sie zeichnen sich alle durch einen grossen Praxisbezug mit einem konkreten Nutzen aus. Seit den 90er Jahren wird die Komplikationenliste in Spitälern geführt, die sich als sehr wertvolles Instrument zur Risikoverminderung bewährt hat [1]. Im ambulanten Bereich werden ebenfalls seit den 90er Jahren Qualitätszirkel gepflegt. Die SGIM hat bereits 2014 die «Smarter Medicine»-Kampagne gestartet, die den Fokus auf vermeidbare Untersuchungen und Behandlungen legt und eine evidenzbasierte
Medizin ins Zentrum stellt [2]. Leitlinien sind ein grosses Thema: Sie sollen die Erkenntnisse der evidenzbasierten Medizin zusammenfassend in der praktischen Arbeit verfügbar machen. In vielen Bereichen sind sie vorhanden, häufig auf internationalen Arbeiten basierend. Die Praxistauglichkeit ist unterschiedlich, häufig sind sie für den Alltag allzu umfangreich.

\section{Konzept}

Alle diese Erkenntnisse sollten dem Patienten nachvollziehbar als Entscheidungshilfe vorliegen, damit eine individuell angepasste Behandlung eingeleitet werden kann. Das Ergebnis muss überprüfbar sein, im Idealfall ist ein Qualitätsindikator daraus abzuleiten. Im Qualitätskonzept der Allgemeinen Inneren Medizin (AIM) werden diese Elemente nun zu einem sinnvollen Ablauf zusammengefügt.

\section{Guidelines}

Die Grundlage für die Qualitätsarbeit bilden die Guidelines, die das gegenwärtig verfügbare Wissen zusam-

\section{Vision «Qualität in der Allgemeinen}

\section{Inneren Medizin»}

\footnotetext{
Die integrierende Funktion der Allgemeinen Inneren Medizin ist gestärkt durch die Zusammenarbeit in Qualitätsthemen mit allen Fachgebieten.

Der generalistische und integrierende Ansatz der Allgemeinen Inneren Medizin setzt ein Gegengewicht zur zuneh menden Fragmentierung der Medizin.

Die Qualitätsarbeit ist fundamental für die Anerkennung der Wichtigkeit der Allgemeinen Inneren Medizin.
} 
menfassen und damit die Basis für die nachfolgenden Entscheidungen bilden. Diese Leitlinien müssen evidenzbasiert sein und das Wissen so aufbereiten, dass es im Alltag einfach verfügbar ist. Idealerweise werden die Kernelemente in einer elektronischen Dokumentation verfügbar gemacht und fliessen nahtlos in die Behandlungsplanung ein. Sie müssen regelmässig aktualisiert werden, damit sie dem aktuellen Stand des Wissens entsprechen. Die internationale Vernetzung mit ähnlichen Initiativen ist zwingend. Die Wissensbasis für diese Leitlinien ist identisch mit derjenigen der «Smarter Medicine»-Kampagne. Die Arbeiten ergänzen sich deshalb ideal.

\section{Anwendung der Guidelines}

Leitlinien beziehen sich auf Krankheiten. Was uns in der Praxis gegenübersitzt, ist nicht eine Krankheit, sondern ein Patient mit seiner spezifischen Persönlichkeit, seiner Geschichte und seinem Umfeld. Eine Behandlung ist letztendlich ein Vertrag mit dem $\mathrm{Pa}$ tienten, der seine individuellen Präferenzen mit einbezieht. Die Guidelines bilden die Wissensbasis, auf deren Grundlage die Meinungsbildung und schliesslich der Entscheid erfolgen soll.

\section{Shared Decision Making und Smarter Medicine}

Das Wissen muss in einem strukturierten Prozess anschaulich und nachvollziehbar vermittelt werden. Dieser Prozess ist als Shared Decision Making beschrieben und ist als tragendes Element im Qualitätskonzept vorhanden [3]. Für die einfache und nachvollziehbare Darstellung von Nutzen und Risiken, wie sie sowohl Ärzte wie Patienten benötigen, ist ebenfalls eine Zusammenarbeit mit etablierten Institutionen anzustreben.

Für das Shared Decision Making kann auf wesentliche bestehende Arbeiten Bezug genommen werden [4]. Eine unverzichtbare Grundlage dafür ist wiederum die einfache und evidenzbasierte Darstellung von Nutzen und Risiko einer Behandlung. Die Ärzte werden geschult in der Interpretation und Vermittlung von statistischen Informationen und in der Kommunikation von Nutzen und Risiken. Insbesondere bei an mehreren Krankheiten leidenden Patienten ist dieser Prozess sehr bedeutsam, da möglicherweise unterschiedliche Leitlinien zur Anwendung kommen und auf einige Massnahmen verzichtet werden muss.

Das «Smarter-Medicine»-Konzept beruht auf den gleichen Grundlagen wie das Shared Decision Making und legt fest, worauf sinnvollerweise verzichtet werden sollte. Beide Elemente ergänzen sich ideal und lassen sich in enger Koordination umsetzen.

\section{Qualitätskonzept «Allgemeine Innere Medizin» Guidelines $\downarrow$ Shared Decision Making - Smarter Medicine $\downarrow$ Zielformulierung $\downarrow$ Zielerreichung}

\section{Behandlungsziel und Zielerreichung}

Mit der Shared Decision lässt sich die Behandlung planen und ein Behandlungsziel formulieren, das den Wünschen und den Ressourcen des Patienten entspricht. Wenn ein klar formuliertes Ziel vorliegt, kann die Zielerreichung gemessen werden.

Damit haben wir einen Qualitätsindikator, der auf der Grundlage der Guidelines die individuelle Situation des Patienten einbezieht und auf den Nutzen für den Patienten fokussiert. Damit kann der Gefahr von abstrakten und nicht situationsgerechten Indikatoren begegnet werden.

\section{Umsetzung}

Die praktische Umsetzung dieses Konzeptes verlangt nach einem multidisziplinären Ansatz, in dem die verschiedenen Fachgebiete für die Erarbeitung der Guidelines einbezogen sein müssen. Eine formale Standardisierung wird deren Verfügbarkeit in einer elektronischen Dokumentation verbessern. Dies kann eine Aufgabe für eine übergeordnete Institution wie die SAQM in Zusammenarbeit mit eHealth sein. Das gesamte Projekt benötigt signifikante Ressourcen, weshalb eine sorgfältige Planung und eine gute Organisation essentiell sind. Hingegen muss nicht mehr jede Institution eigene Leitlinien erarbeiten, was auch ein Sparpotential bedeutet. Die Projektorganisation wird die Arbeiten auf verschiedene Gruppen aufteilen und für ein reibungsloses Zusammenfliessen der Ergebnisse verantwortlich sein.

\section{Literatur}

1 Stäubli M, Suter J. Die Komplikationenliste der Schweizerischen Gesellschaft für Innere Medizin. Schweiz Ärztezeitung. 2004; 85(21):1109-16.

2 Amstad H, Gaspoz JM, Zemp L. Guidelines and Choosing wisely: to do's and not to do's. Schweiz Ärztezeitung. 2015; 96(5): 130-1.

3 Gerber M, Kraft E, Bosshard C. Shared Decision Making - Arzt und Patient entscheiden gemeinsam. Schweiz Ärztezeitung. 2014; 95(50):1883-9.

4 Meier CA. In: Gemeinsam zum Entscheid. Dialog Ethik. TIF 120; April 2015. 